\title{
International journal of machine learning and cybernetics
}

\author{
Xi-Zhao Wang
}

Published online: 11 November 2010

(C) Springer-Verlag 2010

Welcome to the first issue of international journal of machine learning and cybernetics (IJMLC). IJMLC is a refereed academic journal launched by Springer and will publish four printed as well as online issues per year.

It is a great challenge to bring such a new journal into the world. The new journal has a clear focus, the synergy between machine learning and cybernetics. Although there have been several comparable good journals which focus separately on issues in machine learning or cybernetics, there is still much room to synergistically combine the two disciplines. During the recent several decades, the synergy of Machine Learning and Cybernetics is attracting more and more interest in the research community since it promises new, more effective and efficient tools to both machine learning and broadly perceived cybernetics. This synergy has not been explicitly articulated in any other journal we can see today.

IJMLC aims to advance both theoretical and practical developments in Machine Learning and Cybernetics by publishing high quality peer-reviewed articles. In order to achieve this goal, most members in editorial board have been involved in organizing the International Conference on Machine Learning and Cybernetics (ICMLC) for 9 years, i.e., ICMLC2002-ICMLC2010, which were successfully held in different cities. This event has become the leading international gathering of people from academia, professional and industry in the field of Machine Learning and Cybernetics. With this accumulated experience of the editorial board, IJMLC will further promote research in

X.-Z. Wang ( $₫)$

Department of Mathematics and Computer Science,

Hebei University, Hebei, China

e-mail: xizhaowang@ieee.org related issues, and serves as a broad forum for rapid dissemination of the latest advancements.

IJMLC focuses on the hybrid development of machine learning and cybernetics schemes inspired by different contributing disciplines such as engineering, mathematics, cognitive sciences, and applications. New ideas, design alternatives, implementations and case studies pertaining to all the aspects of machine learning and cybernetics fall within the scope of the IJMLC. The topics include supervised learning, unsupervised learning, semi-supervised learning, integrated learning, reinforcement learning, kernel-based Learning, uncertainty processing in the learning, rough techniques in learning, machine learning for modeling interactions between systems, pattern recognition technology to support discovery of system-environment interaction, learning in intelligent control systems, brain and cognitive engineering, system biology, bioinformatics, biochemical interaction in biological and biologically inspired systems, learning for improvement of communication schemes between systems, and fusion of leaning and cybernetics.

All papers published by IJMLC are strictly peer reviewed. In order to ensure high quality of the published materials, IJMLC utilizes a group of experts to review submitted manuscripts. We do our best to select the most appropriate referees for a manuscript while at the same time making sure they are not overburdened with reviews. The associate editors play a critical role in helping us to manage the review process of manuscripts. I am honored to have 20 associate editors and 11 advisory board members from more than 10 countries, who are active researchers committed to excellence in research in machine learning and cybernetics. Without the service and dedication of our editorial board, IJMLC would have never existed.

After the rigorous review process, this inaugural issue includes seven manuscripts. 
Kevin Small's article, Margin-based active learning for structured predictions, extends the application scope of existing margin-based active learning method, especially in complex prediction problems which require combining multiple prediction results or decomposing a complex prediction into a sequence of predictions.

Battista Biggio's paper, Multiple classifier systems for robust classifier design in adversarial environments, deals with the issue of classifier ensembles in adversarial classification tasks. Its focus is to improve the robustness of linear classifiers with adversarial data, as well as study experimentally the utility of bagging and random space method to implement these classifiers.

Authored by Yin Zhang, Rong Jin and Zhi-Hua Zhou, the paper Understanding bag-of-words model: a statistical framework, explores theoretical studies on properties of the bag-of-words model, which remains untouched in the literature. The most important contribution of this paper is the development of a statistical framework which generalizes the bag-of-word representation. Theoretical analysis is presented and then two algorithms are proposed based on this framework. The competitive experimental results are also given for object categorization compared with clustering-based bag-of-word representations.

Ludmila I. Kuncheva's article, Full-class set classification using the Hungarian algorithm, proposes a Bayesoptimal method to full-class set classification problems, which has only one object coming from each class. The merit of this method is demonstrated by both theoretical and experimental results.

Qinghua Hu's article, An efficient gene selection technique for cancer recognition based on neighborhood mutual information, introduces the concept of neighborhood mutual information as a measure to describe the relevance of genes and related decisions. A gene selection algorithm is then constructed by combining this measure with the search strategy of minimal redundancy and maximal relevancy. Finally, the number of genes can be further reduced to provide an efficient gene selection algorithm.

Dong Ling Tong's paper, Genetic Algorithm-Neural Network (GANN): a study of neural network activation functions and depth of genetic algorithm search applied to feature selection, investigates the use of hybrid GA and ANN as a feature selection method, and studies the impact of ANN activation function and the number of GA evaluations on feature selection performance.

Nita H. Shah's paper, Optimal production schedule in declining market for an imperfect production system, aims to develop an inventory minimization model when there is a certain percent of product is of imperfect quality, which is an issue often emerging in economic production. The model is validated by numerical examples and sensitivity analysis.

I would like to take this opportunity to deliver my sincere thanks to those who contribute to the process of establishment and running of this journal. Thanks first to Prof. Daniel Yeung and Prof. Witold Pedrycz for their fundamental contribution while establishing this journal. Thanks to our advisory board members who have given very valuable suggestions and comments for establishing and running this journal. Thanks to our associate editors for their time and effort in effectively handling the review process. Thanks also to our referees for the time and effort spent in reviewing manuscripts to ensure only high quality manuscripts are selected for publication in IJMLC.

Additionally I would like to thank my fellows in Springer working together for publishing IJMLC. Last but not least, let me thank many authors who have submitted manuscripts to IJMLC for review. I hope that they will continuously support IJMLC and also encourage more colleagues to submit quality manuscripts. 